\title{
Genetic parameters and responses of performance and body composition traits in pigs selected for high and low growth rate on a fixed ration over a set time
}

\author{
Nguyen Hong NGUYEN ${ }^{\mathrm{a} *}$, Cameron P. MCPHEE ${ }^{\mathrm{b}}$ \\ ${ }^{a}$ School of Veterinary Science, University of Queensland, QLD 4072, Australia \\ ${ }^{b}$ Animal Research Institute, Queensland Department of Primary Industries, Australia
}

(Received 26 May 2004; accepted 11 October 2004)

\begin{abstract}
Two lines of Large White pigs of common genetic origin were divergently selected over four years for high and low growth rate during a 6 week post-weaning test period in which all pigs were fed the same total amount of food ( $80 \%$ of estimated ad libitum intake). Genetic parameters and direct and correlated responses in performance and carcass traits were estimated on 2884 pigs with pedigrees comprising a total of 5324 animals, with restricted maximum likelihood and best linear unbiased prediction methods applied to a multi-trait animal model. Estimates of heritability $( \pm \mathrm{SE})$ were $0.19 \pm 0.04$ for lifetime daily gain (LDG), $0.16 \pm 0.03$ for test daily gain (TDG), $0.25 \pm 0.04$ for ultrasound P2 backfat (UBF) and $0.16 \pm 0.03$ for food conversion ratio during test (TFC), and $0.15 \pm 0.04$ for daily carcass weight gain (CDG), $0.43 \pm 0.06$ for carcass backfat (CFT) and $0.40 \pm 0.06$ for carcass lean percentage (LEAN). Common litter effects for TDG, UBF and TFC were less than 5\% and for LDG, $17 \%$ of total phenotypic variance. Genetic correlations between performance and carcass traits were moderately to highly favourable. After four years of divergent selection for growth rate, the selection responses in estimated breeding value (EBV) for TDG were 40.14 and $-41.11 \mathrm{~g}$ (SED 2.95) for the high and low growth lines, respectively. The regressions of EBV on year of birth, indicate that the annual genetic trend for TDG, was $8.73 \mathrm{~g} / \mathrm{yr}$ in the high and $-8.48 \mathrm{~g} / \mathrm{yr}$ in the low lines $(P<0.001)$. Correlated genetic responses in the high and low lines respectively were $5.28 \mathrm{~g}$ and $-12.40 \mathrm{~g}$ (SED 2.09) in LDG, $-0.35 \mathrm{~mm}$ and $0.56 \mathrm{~mm}$ (SED 0.009) in UBF, -0.145 units and 0.185 units (SED 0.012) in TFC, $3.17 \mathrm{~g}$ and $-10.97 \mathrm{~g}$ (SED 1.53) in CDG, $-1.13 \mathrm{~mm}$ and $1.01 \mathrm{~mm}$ (SED 0.155 ) in CFT and $1.24 \%$ and $-1.27 \%$ (SED 0.150) in LEAN. It was concluded that selection for increased post-weaning daily gain on a ration of fixed amount reduces the age at slaughter and the level of backfat and increases the efficiency of food utilisation, weight and leanness of pig carcasses.
\end{abstract}

divergent selection / pig / growth / restricted feeding / genetic responses

${ }^{*}$ Corresponding author: nhongnguyen@yahoo.com Permanent address: Institute of Agricultural Science of South Vietnam, 121 Nguyen Binh Khiem Street, District 1, HoChiMinh City, Vietnam 


\section{INTRODUCTION}

The characteristics of the pig that are positive for profitability are high growth rate, low food conversion ratio and low fatness of the carcass [19]. A profitable pig is one which, from each unit of food energy, wastes least in such processes as digestion and physical activity and retains most by favouring the more efficient conversion of metabolisable energy to lean than the less efficient conversion to fat tissue [25]. It is hypothesised that such a pig would reveal itself by growing more quickly than its contemporaries when all are given the same ration of food over a feeding period of fixed weight [15] or given duration [23]. Genetic variation in such a characteristic could be the raw material of a genetic selection programme to produce a more profitable pig. Selection for high lean growth rate on a scale feeding [7] has been shown to improve both the rate and efficiency of lean growth, by giving the benefits but not the disadvantages of selection for lean food conversion and lean growth rate on ad libitum feeding $[4,21]$. However, selection strategies emphasising high leanness was inferred to raise metabolic heat production and the associated rate of glycogen depletion with fasting as measured by muscle acidity [20]. This study sets out to test a hypothesis of improving lean conversion efficiency without increasing metabolic rate by forming lines selected for high and for low growth rate while on a restricted feeding scale of fixed total amount during a post-weaning performance test of set duration. Selection responses are measured on both scale and ad libitum feeding for growth rate, carcass fatness, voluntary food intake and food conversion efficiency. This paper reports genetic parameters and responses in growth and carcass traits in the lines, measured on the restricted feeding scale over four years.

\section{MATERIALS AND METHODS}

\subsection{Establishment of selection lines}

The present selection lines originated from a continuing series of selection experiments in pigs for high efficiency of lean growth over the past 25 years $[18,21]$. For this study, a new base population was formed by outcrossing sows from the selected lines with superior Large White boars identified in a boar performance testing station. DNA testing of the original base population stocks ensured that the herd was free from the halothane gene. 
Two lines were established by sampling within litters, a high line selected for high growth rate and a low line selected for low growth rate. Each line consisted of 6 boars and 36 sows. Within a line, three batches were formed each with 12 sows of the high line and 12 sows of the low line. The matings of sows within each batch were synchronised and there was a period of 6.5 weeks between batches. The first litter for selection was produced in early 1997. At five weeks of age, the litters were weaned, and after reaching $50 \mathrm{~kg}$ body weight, performance testing was carried out for 6 weeks and selection of breeding replacements was then made. This pattern of production, testing and selection was followed for the four-year duration of the experiment.

\subsection{Pre-test management of pigs}

At five weeks of age, the piglets were weaned and transferred to the weaner shed of the farrowing/weaner building where they remained until ten weeks of age in groups of 10 to 13 per $2.1 \times 2.1 \mathrm{~m}$ pen or in groups of 20 to 26 per $4.4 \times 2.2 \mathrm{~m}$ pen. A commercial weaner diet with $14.8 \mathrm{MJ} \mathrm{DE} / \mathrm{kg}$ and $0.75 \mathrm{~g}$ available lysine per MJ DE was provided ad libitum, and water with nipple drinkers of approved pattern. After reaching approximately $30 \mathrm{~kg}$ live weight, the pigs from both lines were intermingled and reared in groups of 20-30 pigs of similar weight and sex in $8 \times 16 \mathrm{~m}$ pens. During this period, they were fed ad libitum on a moist diet of $14.1 \mathrm{MJ} \mathrm{DE} / \mathrm{kg}$ and $0.64 \mathrm{~g}$ available lysine per MJ $\mathrm{DE}$ to $50 \mathrm{~kg}$ (at about 27 weeks of age), the weight at the start of performance testing.

\subsection{Performance testing and selection procedures}

Following the attainment of $50 \mathrm{~kg}$ live weight $(\mathrm{CV}=2.59 \%)$, each batch (since 1997) of 24 male and 24 female pigs from the high and low growth selection line were placed in groups of six and fed once a day in individual feeding stalls. The pigs were all fed the same total amount of grower food over a six-week testing period, restricted to approximately $80 \%$ of average ad libitum intake. The levels of feed restriction were determined from a trial on unselected pigs fed ad libitum from 25 to $90 \mathrm{~kg}$ in the base generation, one for the winter with $85 \%$ of the ad libitum trial and one for the summer with $90 \%$ of the winter scale [19]. Food residues remaining after a few hours of feeding were collected, and oven dried and weighed, and an equivalent amount was fed back on subsequent days. In the summer, the daily allowance was $1.85 \mathrm{~kg}$ in the first week and this increased by approximately $0.10 \mathrm{~kg}$ each week to reach 
$2.20 \mathrm{~kg}$ in the sixth week. In the winter, the amount of daily allowance was $2.05,2.1,2.2,2.25,2.30,2.35 \mathrm{~kg} / \mathrm{d}$ for weeks one to six. The diet comprised wheat, soya bean, fish meal, minerals and vitamins and contained 14.1 MJ DE, $0.65 \mathrm{~g}$ available lysine per MJ DE. The live weights recorded at the start and end of the test were used to calculate the test average daily gain (TDG). Lifetime average daily gain (LDG) was computed as the ratio of the end weight to days from birth to slaughter. Food conversion ratio on test (TFC) was the ratio of average daily food intake to TDG. Backfat thickness (UBF) was ultrasonically measured at the $\mathrm{P} 2$ position at the end of the test.

At the end of the six-week performance testing period with an average body weight of $80 \mathrm{~kg}$, the 24 boars and 24 gilts tested per batch in each selection line were ranked on growth rate on test, and two boars and six gilts were selected as breeder replacements per batch per line. In this way there were 12 males and 36 females entering each line annually so that the sows were replaced after three farrowings and boars after six matings. However, some sows were kept for more than three farrowings before culling when there were too few mated gilt replacements available. Additional boars and sows were reserved as spares in cases of death loss or breeding failure.

At the end of the test, the pigs were also tattooed and transported on an $8 \mathrm{~h}$ journey from their grow-out farm in Central Queensland to an abattoir in Brisbane where they were lairaged overnight without food but with water. Hot carcass weight (HCW) and carcass fat depth (CFT) were measured at slaughter. Measurement of fat was made at the P2 position using a Hennessy Grading Probe (Hennessy Grading Systems, Auckland, New Zealand). Hot carcass weight included fore and hind trotters, and leaf fat but not the head. Average daily carcass gain (CDG) was calculated from the hot carcass weight over days from birth to slaughter. Predicted lean meat percentage (LEAN) was based on adjusted hot carcass weight and carcass fat measurements [6].

\subsection{Data characteristics}

Out of a total of 5324 animals born in the herd with full pedigrees recorded, 2884 pigs from 831 litters in 33 batches had measurements taken of performance on restricted feeding from the start in February 1996 to the end of the experiment in December 2000. Carcass composition traits were also measured on 1746 animals from the lines which had been divergently selected for high or low growth rate. They were the progeny of 121 sires and 367 dams. 


\subsection{Statistical analysis}

\subsubsection{Testing of fixed and random effects}

Preliminary analyses using the general linear model (GLM) procedure were first performed to determine the significance of the fixed effects and covariates [9] where the conditional F-tests are implemented in the form of the ANOVA method. The significance of different nested random terms in models was assessed based on both the ratios of variance components to standard errors and likelihood ratio test, using ASREML [10]. The variance component estimates of common litter effects for LDG, TDG, UBF and TFC were all greater than 2.0 standard errors (Component/Standard error $>2.0$ ), indicating the effects to be significant [10]. These ratios were 1.7 for CDG, 1.6 for CFT and 1.1 for LEAN, which are questionably significant. A likelihood ratio test comparing twice the difference in logarithmic likelihoods between the full and reduced models with chi-square $\left(\chi^{2}\right)$ having degrees of freedom equal to the number of parameters tested, was then tested and it was verified that the effects of common litter were not significant for any carcass composition trait $(P>0.05)$. Maternal genetic effects were tested in a similar manner to be insignificant for all traits and were therefore excluded from the genetic models of analysis.

\subsubsection{Estimation of genetic parameters}

All analyses for genetic parameters were carried out with ASREML combining the average information algorithm and sparse matrix methods to give an efficient algorithm in both computing time and working space [10]. The method enabled the inclusion of different fixed effects, covariates and random effects in the model for each trait (Tab. I). The fixed effects of batch (33 classes) and sex ( 2 classes) were fitted for both performance and carcass composition traits, but different covariates (AGE and $\mathrm{HCW}$ ) were fitted for the latter traits. Common environmental effects were included for the performance test traits only, but not for the carcass traits, since they were tested to be insignificant for these traits in the model. Generally univariate analyses were carried out first to obtain genetic and environmental variances for all traits. A series of bivariate model analyses was then run to estimate covariance components, which were subsequently applied to multi-trait models to derive genetic and phenotypic correlations among the traits. 
Table I. Model of analysis and significance levels of fixed effects.

\begin{tabular}{|c|c|c|c|c|c|c|}
\hline \multirow[t]{2}{*}{ Traits } & \multicolumn{2}{|c|}{ Fixed effects } & \multicolumn{2}{|c|}{ Covariates } & \multicolumn{2}{|c|}{ Random effects } \\
\hline & Batch & Sex & AGE & $\mathrm{HCW}$ & Animal & Common litter \\
\hline \multicolumn{7}{|c|}{ Production traits } \\
\hline LDG & $* * *$ & $* *$ & & & $\checkmark$ & $\checkmark$ \\
\hline TDG & $* * *$ & $* * *$ & & & $\checkmark$ & $\checkmark$ \\
\hline UBF & $* * *$ & $* *$ & & & $\checkmark$ & $\checkmark$ \\
\hline TFC & $* * *$ & $* * *$ & & & $\checkmark$ & $\checkmark$ \\
\hline \multicolumn{7}{|c|}{ Carcass traits } \\
\hline CDG & $* * *$ & $* * *$ & & & $\checkmark$ & ns \\
\hline CFT & $* * *$ & $* * *$ & $*$ & $* * *$ & $\checkmark$ & ns \\
\hline LEAN & $* * *$ & $* * *$ & $* *$ & & $\checkmark$ & ns \\
\hline
\end{tabular}

*** $P<0.001 ; * * P<0.01 ; * P<0.05$; ns: non-significant.

\subsubsection{Prediction of breeding values}

Breeding values for all the traits were estimated with the best linear unbiased prediction (BLUP) analysis of the PEST package [11]. The additive genetic and residual (co) variances used in the multivariate model included the same fixed and random effects as described in the estimation of genetic parameters. In the analysis, test average daily gain (TDG) was the trait that the animals had been selected for and LDG, UBF and TFC, and CDG, CFT and LEAN were included to avoid selection biases. The means of EBV for all traits were derived for the high and low growth lines for each year of selection and genetic trends were estimated by regression of individual EBV on year of birth [9].

\section{RESULTS AND DISCUSSION}

\subsection{Means and standard deviations}

Means, standard deviations and coefficients of variation for lifetime daily gain (LDG), test daily gain (TDG), ultrasonic P2 backfat (UBF), food conversion ratio (TFC), carcass weight daily gain (CDG), carcass fat (CFT) and predicted lean percentage (LEAN) are given in Table II.

\subsection{Phenotypic responses}

Selection line phenotypic means for TDG, LDG, UBF and TFC, and CDG, CFT and LEAN at the conclusion of the selection are presented in Table III. 
Table II. Number of records $(N)$, mean, standard deviation of the means (SD), and coefficients of variation (CV \%) for performance test and carcass traits.

\begin{tabular}{llllll}
\hline Traits & Unit & $N$ & Means & SD & CV \\
\hline Production traits & & & & & \\
LDG & g/d & 2884 & 546 & 54.6 & 10.0 \\
TDG & g/d & 2884 & 739 & 86.7 & 11.7 \\
UBF & mm & 2884 & 11.9 & 1.9 & 17.0 \\
TFC & unit & 2884 & 2.9 & 0.4 & 13 \\
Carcass traits & & & & & \\
CDG & g/d & 1746 & 450.2 & 51.4 & 11.4 \\
CFT & mm & 1746 & 15.9 & 4.0 & 25.3 \\
LEAN & $\%$ & 1746 & 57.3 & 3.8 & 6.6 \\
\hline
\end{tabular}

Table III. Selection line phenotypic means for performance traits on restricted feeding for the high and low growth lines after four years of selection.

\begin{tabular}{lcclll}
\hline Traits & HL & LL & HL - LL & S.E.D. & Significance \\
\hline Production traits & & & & & \\
LDG (g) & 512.7 & 500.4 & 12.3 & 4.319 & $P<0.001$ \\
TDG (g) & 773.0 & 692.1 & 80.9 & 7.997 & $P<0.001$ \\
UBF (mm) & 11.46 & 12.44 & -0.98 & 0.205 & $P<0.001$ \\
TFC (unit) & 2.835 & 3.172 & -0.34 & 0.034 & $P<0.001$ \\
Carcass traits & & & & & \\
CDG (g) & 438.6 & 423.9 & 14.7 & 5.216 & $P<0.05$ \\
CFT (mm) & 14.76 & 16.75 & -1.99 & 0.459 & $P<0.01$ \\
LEAN (\%) & 58.54 & 56.08 & 2.46 & 0.434 & $P<0.001$ \\
\hline
\end{tabular}

S.E.D.: standard error of difference between the high (HL) and low (LL) selection lines; HL - LL: the difference between high and the low selection lines.

There were significant differences $(P<0.05)$ between the selection lines for all the observed traits after four years of selection although the actual responses fluctuated from year to year in each line due to sampling effects. Some variation, common to both lines, may have been due to changes in diet ingredients caused by a shortage of wheat, leading to a slight deterioration in TDG and TFC between 1998 and 1999.

\subsection{Genetic parameters}

\subsubsection{Heritabilities and common litter effects}

The current estimates of heritability for production and carcass traits in pigs subject to a 6-week post-weaning test period on restricted feeding were mostly 
moderate (Tab. IV), and fell within the published ranges of the literature reviewed by Clutter and Brascamp [5] and Nguyen [24]. Because the DNA test of the original base population assured that the lines of the present study were halothane gene-negative, the estimates of heritability for all traits were free of bias from this gene. Although common litter effects, tested by the likelihood ratios or the ratios of variance components to their standard errors, were significant for all traits, they accounted for only a small proportion of the total phenotypic variances (5\% for TDG, UBF and TFC), except for LDG (17\%). The large effect of common litter for LDG relative to TDG and other traits indicates that resemblance between sibs is due to shared environments as well as shared genes, reflecting variation in mothering ability among dams, e.g. differences in milk production capacity leading to differences between litters in early growth, a component of LDG but not TDG. In other populations fed either on a restricted or semi-restricted scale, the common litter effects were found to range from -3 to $28 \%$ for ADG, 32 to $42 \%$ for ages to $100 \mathrm{~kg},-6$ to $26 \%$ for UBF and 13 to $19 \%$ for TFC [3, 4,13,17,22]. Variances due to common litter effects were not significantly greater than zero for any carcass composition traits, in agreement with the estimates reported by Lundeheim et al. [17], Merks [22] and Johansson et al. [13].

\subsubsection{Genetic correlations}

Genetic correlations among performance traits estimated under restricted feeding of this current study (Tab. IV) were all economically favourable, consistent with the published estimates of -0.07 to -0.33 for TDG and UBF, -0.93 to -1.07 for TDG and TFC, and 0.16 to 0.30 for UBF and TFC [5, 24]. The directions of common environmental correlations for litter effects among the traits (Tab. V) were similar to those of the genetic correlations.

The moderate, negative genetic correlations of LDG and TDG with UBF $(-0.25$ and -0.39$)$ suggest that selection for increased growth rate under a ration constraint, would exploit genetic variation in the partitioning of energy towards lean growth and away from fat deposition presumably because of the higher energy cost required to deposit the latter tissue than the former one [25]. Levels of food restriction have been shown by others to influence the sign and magnitude of the genetic correlation between growth rate and backfat thickness $[1,12,21]$. At both the genetic and phenotypic levels, the correlations between TDG and TFC are close to minus one, reflecting a low variation $(\mathrm{CV}=2.1 \%)$ between animals in food intake during the test. In addition, the genetic correlation between UBF and TFC was highly positive 
Table IV. Heritabilities (bold), and phenotypic (above diagonal) and genetic correlations (below diagonal) respectively for performance and carcass traits on restricted feeding.

\begin{tabular}{llllllll}
\hline Traits & \multicolumn{1}{l}{ LDG } & \multicolumn{1}{l}{ TDG } & \multicolumn{1}{l}{ UBF } & \multicolumn{1}{l}{ TFC } & \multicolumn{1}{l}{ CDG } & \multicolumn{1}{l}{ CFT } & \multicolumn{1}{l}{ LEAN } \\
\hline LDG & $\mathbf{0 . 1 9} \pm \mathbf{0 . 0 4}$ & $0.46 \pm 0.02$ & $-0.04 \pm 0.02$ & $-0.41 \pm 0.02$ & $0.44 \pm 0.14$ & $-0.15 \pm 0.03$ & $0.09 \pm 0.03$ \\
TDG & $0.60 \pm 0.11$ & $\mathbf{0 . 1 6} \pm \mathbf{0 . 0 3}$ & $-0.02 \pm 0.02$ & $-0.94 \pm 0.00$ & $0.26 \pm 0.03$ & $-0.12 \pm 0.02$ & $0.09 \pm 0.03$ \\
UBF & $-0.25 \pm 0.15$ & $-0.39 \pm 0.14$ & $\mathbf{0 . 2 5} \pm \mathbf{0 . 0 4}$ & $0.02 \pm 0.02$ & $0.05 \pm 0.02$ & $0.31 \pm 0.03$ & $-0.32 \pm 0.03$ \\
TFC & $-0.55 \pm 0.13$ & $-0.99 \pm 0.00$ & $0.43 \pm 0.13$ & $\mathbf{0 . 1 6} \pm \mathbf{0 . 0 3}$ & $-0.24 \pm 0.02$ & $0.13 \pm 0.03$ & $-0.09 \pm 0.03$ \\
CDG & $0.86 \pm 0.08$ & $0.44 \pm 0.14$ & $-0.12 \pm 0.16$ & $-0.42 \pm 0.15$ & $\mathbf{0 . 1 5} \pm \mathbf{0 . 0 4}$ & $0.26 \pm 0.03$ & $-0.24 \pm 0.03$ \\
CFT & $-0.65 \pm 0.12$ & $-0.56 \pm 0.12$ & $0.81 \pm 0.07$ & $0.58 \pm 0.12$ & $-0.22 \pm 0.15$ & $\mathbf{0 . 4 3} \pm \mathbf{0 . 0 6}$ & $-0.58 \pm 0.07$ \\
LEAN & $0.56 \pm 0.14$ & $0.50 \pm 0.13$ & $-0.82 \pm 0.07$ & $-0.53 \pm 0.12$ & $0.19 \pm 0.17$ & $-0.57 \pm 0.08$ & $\mathbf{0 . 4 0} \pm \mathbf{0 . 0 5}$ \\
\hline
\end{tabular}


Table V. Common litter effects (bold), residual (above diagonal) and common litter correlations (below diagonal) respectively for performance traits on restricted feeding.

\begin{tabular}{llllr}
\hline Traits & \multicolumn{1}{l}{ LDG } & \multicolumn{1}{l}{ TDG } & UBF & \multicolumn{1}{l}{ TFC } \\
\hline LDG & $\mathbf{0 . 1 7} \pm \mathbf{0 . 0 3}$ & $0.48 \pm 0.02$ & $0.11 \pm 0.02$ & $-0.41 \pm 0.03$ \\
TDG & $0.15 \pm 0.16$ & $\mathbf{0 . 0 4} \pm \mathbf{0 . 0 2}$ & $0.10 \pm 0.02$ & $-0.96 \pm 0.00$ \\
UBF & $-0.24 \pm 0.15$ & $-0.18 \pm 0.26$ & $\mathbf{0 . 0 5} \pm \mathbf{0 . 0 2}$ & $-0.10 \pm 0.02$ \\
TFC & $-0.22 \pm 0.15$ & $-0.95 \pm 0.08$ & $0.12 \pm 0.25$ & $\mathbf{0 . 0 5} \pm \mathbf{0 . 0 2}$ \\
\hline
\end{tabular}

(0.41), indicating that selection for high growth rate, low backfat, or joint selection for increased growth and reduced backfat, would result in significant improvement in lean tissue food conversion efficiency. As market targets for fatness are satisfied, the goal of pig selection for improving efficiency should be directed to reducing the amount of energy used for basal heat production and to increasing the efficiency of energy partitioning between lean and fat tissue growth. Selection for reduced lean food conversion ratio on ad libitum feeding has been shown to place major emphasis on reducing food intake, the numerator of the ratio and only a minor emphasis on increasing growth rate, the denominator of the ratio [4,21]. Restricted feeding appears preferable to $\mathrm{ad}$ libitum feeding as a performance testing regimen for improving the ratio since the variation in food intake is suppressed, diverting the selection effort to lean growth. However, there is still a trend of reducing voluntary food intake under a restricted test system [7], e.g. the portion associated with maintenance, i.e., not associated with growth [24].

Genetic correlations among carcass traits were negative between CDG and CFT, and positive between CDG and LEAN, indicating that all these three traits could be easily improved by selection simultaneously. This is in good agreement with the results reported in Dutch and Swedish pigs also tested on restricted feeding $[14,22]$.

The genetic correlations of LDG and TDG were strongly positive with CDG and LEAN and highly negative with CFT, suggesting that selection for growth rate on restricted feeding would increase slaughter weight, dressing percentage and lean content, and also decrease fatness in the carcasses of the animals. It follows that the efficiency of lean production on restricted feeding would also be improved through the favourable genetic correlations of food conversion ratio on testing (TFC) with CFT and LEAN, respectively. As expected from the fixed rations fed during performance testing, the genetic correlations of TFC were opposite in sign but of similar magnitude to those of TDG with CDG, CFT and LEAN. The use of ultrasonic backfat (UBF) would be an effective selection criterion to improve carcass quality since the genetic correlations of 
ultrasonic measurement of backfat (UBF) on the live pigs at the end of the test with carcass fat (CFT) using the Hennessy Grading Probe and LEAN were highly positive $(0.81)$ and negative $(-0.82)$, respectively. A reduction in UBF would also be expected to increase the slaughter weight of the animals.

\subsection{Direct and correlated responses}

Genetic changes measured as estimated breeding values (EBV) from multitrait BLUP analyses for all measured traits over the four years of selection are presented in Figures 1a-1f. Substantial response was achieved through selection for test growth rate on restricted feeding, with a divergence between the high and low growth lines in the genetic mean of daily gain by $81.25 \mathrm{~g} / \mathrm{d}$ or 2.78 genetic standard deviations after four years of selection. The divergence in TDG, particularly at the conclusion of the selective process was symmetrical (40.14 vs. $-41.11 \mathrm{~g} / \mathrm{d})$. The regressions of individual EBV on years of birth gave annual genetic improvements in TDG of 8.73 and $-8.48 \mathrm{~g} / \mathrm{year}$ in the high and low growth lines, respectively. The symmetry of response arose from similar selection proportions applied to both lines, with averages of $19 \%$ and $29 \%$ respectively of the contemporaneously available tested boars and gilts selected in each line. The average rates of inbreeding were also found to be similar in both selection lines ( $8.69 \%$ in the high vs. $7.89 \%$ in the low growth line).

Selection was for fast and low growth only in the present study, but gains occurred in other economically important traits. This resulted from the highly favourable genetic correlations of growth rate with ultrasonic backfat (UBF), food conversion ratio (TFC), carcass weight daily gain (CDG), carcass fat $(\mathrm{CFT})$ and lean percentage (LEAN). The achieved responses here were comparable with increases of 12.6 to $25.4 \mathrm{~g} / \mathrm{d}$ for TDG, and decreases of -0.46 to $-0.70 \mathrm{~mm}$ for UBF and -0.05 unit for TFC per generation of selection for a more sophisticated selection criterion (lean growth rate), with pigs also tested on restricted feeding [2,21]. Literature reports of phenotypic changes in backfat in response to selection for increased growth rate in pigs fed ad libitum were either favourable [8] or unfavourable $[16,26]$. To fully measure the hypothesised changes from growth selection on restricted feeding, it is necessary to measure changes in voluntary food intake and this was done by growing pigs sampled from the lines on ad libitum feeding. The results of this are reported in a later paper.

The central aspect of the selection procedure in the present study was the requirement that all pigs of a batch consume the same amount of food in the six-week testing period. Removing food intake as a contributor to variation in 


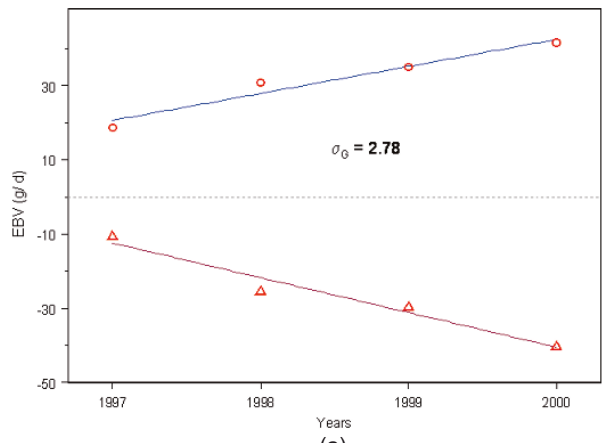

(a)

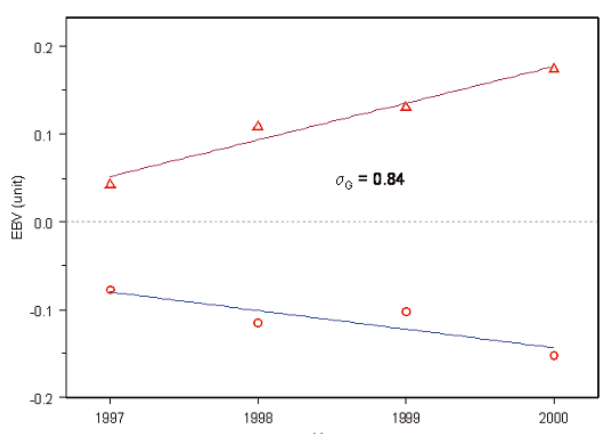

Years
(d)

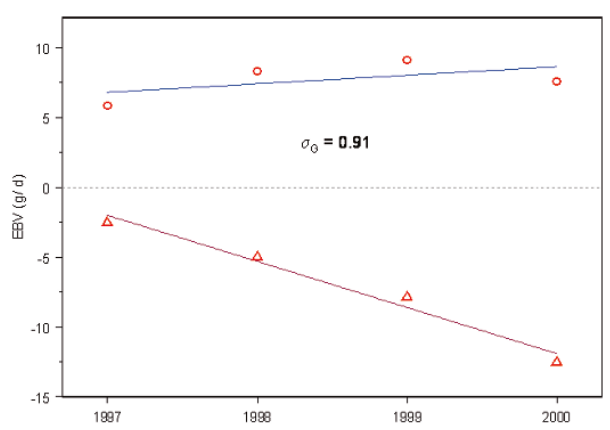

(b)

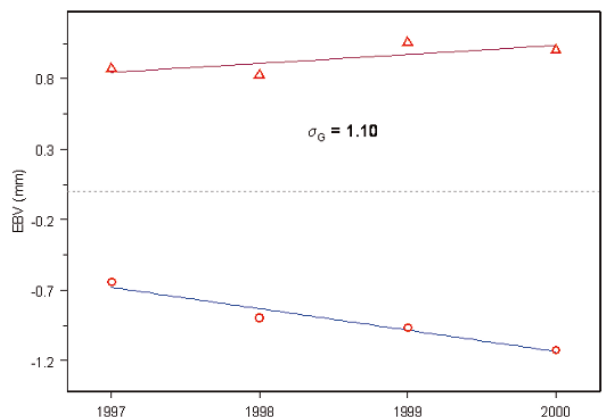

Years
(e)

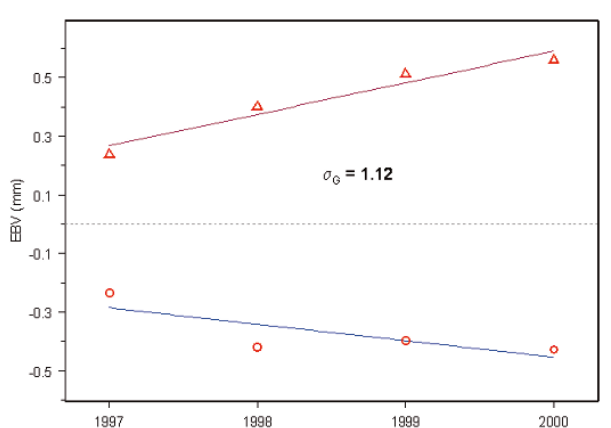

Years
(c)

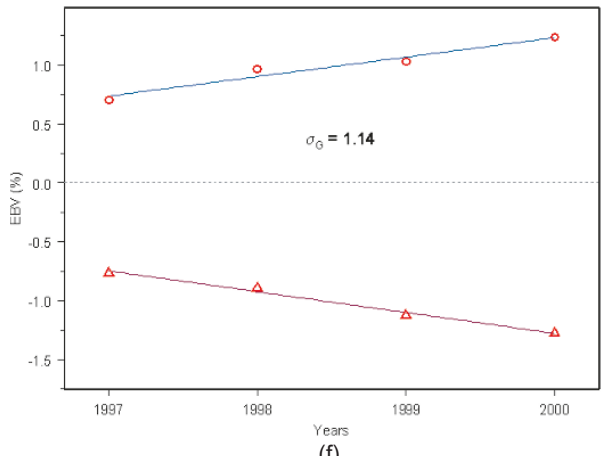

Years
(f)

Figure 1. Estimated breeding values (EBV) for test daily gain (a), lifetime daily gain (b), ultrasonic backfat (c), test food conversion ratio (d), carcass fat (e), and carcass lean percentage (f) over the four years of selection. $\sigma_{\mathrm{G}}$ : divergences in genetic standard deviations between the high $(\circ)$ and low $(\triangle)$ growth lines. 
growth rate in this way ensured that a maximum selection pressure was placed on the efficiency of gain. The extent to which variation in food intake was controlled is exemplified in the low CV (2.1\%) achieved for this trait. Part of the control was achieved through the practice of measuring any food residues that an animal might leave in a particular day then feeding this back in subsequent days during the test period. With the continuing development of computerised electronic feeding, the automation of this feeding system becomes increasingly feasible.

It is summarised that selection for growth rate over a 6-week post-weaning testing period on a fixed but restricted ration significantly improved growth, efficiency and carcass leanness of the animals when measured on the same feeding regimen. The genetic trends for the observed traits diverged symmetrically between the selection lines. Discriminant analysis of EBV of animals born in 2000 for the traits also indicated that after four years of selection the two lines have diverged sufficiently to allow the correct classification into the high $(96.1 \%)$ and low $(97.3 \%)$ growth lines. These genetically diverse lines are therefore suitable for use for the subsequent detailed study of biological and physiological changes brought about by the selection criterion used.

\section{CONCLUSIONS}

Selection for post-weaning daily gain on restricted feeding significantly increased the rate of growth, the weight and percentage of lean in the carcasses, and decreased backfats and food conversion ratio. These changes resulted from genetic correlations which were highly positive between growth rate and CDG and LEAN, and highly negative between growth rate and CFT and TFC. It is concluded that selection for increased growth rate on restricted feeding would increase both the efficiency with which food is converted to lean production and the rate of lean growth and would reduce the rate of fat growth.

\section{ACKNOWLEDGEMENTS}

This project was funded in part by the Australian Center for International Agricultural Research. Mr. Lindsay Daniels is acknowledged for the animal management and assistance in data collection. 


\section{REFERENCES}

[1] Cameron N.D., Curran M.K., Selection for components of efficient lean growth rate in pigs. 4. Genetic and phenotypic parameter estimates and correlated responses in performance test traits with ad libitum feeding, Anim. Prod. 59 (1994) 281-291.

[2] Cameron N.D., Curran M.K., Responses in carcass composition to divergent selection for components of efficient lean growth rate in pigs, Anim. Sci. 61 (1995) 347-359.

[3] Cameron N.D., Curran M.K., Thompson R., Estimation of sire with feeding regime interaction in pigs, Anim. Prod. 46 (1988) 87-95.

[4] Cameron N.D., Curran M.K., Kerr J.C., Selection for components of efficient lean growth rate in pigs. 3. Responses to selection with a restricted feeding regime, Anim. Prod. 59 (1994) 271-279.

[5] Clutter A.C., Brascamp E.W., Genetics of performance traits, in: Rothschild M.F., Ruvinsky A. (Eds.), The genetics of the pig, CAB International, Wallingford, UK, 1998, pp. 427-462.

[6] Ferguson D.M., Chandler R.C., Mynard P., Thomas M., The validation of equations for the prediction of lean meat yield in pig carcasses, PDRC LMA6.P final report, 1994.

[7] Fowler S.H., Bichard M., Pease A., Objectives in pig breeding, Anim. Prod. 23 (1976) 365-387.

[8] Fredeen H.T., Mikami H., Mass selection in a pig population: correlated changes in carcass merit, J. Anim. Sci. 62 (1986) 1546-1554.

[9] GenStat 6. Release 6.1 for Windows, Sixth Edition, VSN International Ltd., Oxford, UK, 2002.

[10] Gilmour A.R., Cullis B.R., Welham S.J., Thompson R., ASREML Reference Manual, NSW Agriculture Biometric Bulletin $N^{\circ} 3$, Orange Agricultural Institute, Forest Road, Orange 2800 NSW Australia, 1999.

[11] Groeneveld E., PEST user's Manual, Institute of Animal Husbandry and Animal Behaviour, Federal Agricultural Research Centre, Germany, 1990.

[12] Gu Y., Haley C.S., Thompson R., Estimates of genetic and phenotypic parameters of growth and carcass traits from closed lines of pigs on restricted feeding, Anim. Prod. 49 (1989) 467-475.

[13] Johansson K., Andersson K., Lundeheim N., Evaluation of station testing of pigs. I. Genetic parameters for feed measurements and selection effects on voluntary feed intake, Acta Agric. Scand. 37 (1987) 93-107.

[14] Johansson K., Andersson K., Sigvardsson J., Evaluation of station testing of pigs. III. Genetic parameters for carcass measurements of partially dissected pigs, Acta Agric. Scand. 37 (1987) 120-129.

[15] Kielanowski J., The method of pig progeny testing applied, in: Proceeding of meeting of the sub-commission on pig progeny testing, 19th annual meeting of the European Association for Animal Production, 1968, Dublin, Ireland.

[16] Kuhlers D.L., Jungst S.B., Mass selection for increased 200-day weight in a closed line of Landrace pigs, J. Anim. Sci. 69 (1991) 977-984. 
[17] Lundeheim N., Johansson K., Andersson K., Estimated phenotypic and genetic parameters based on data from the Swedish pig progeny testing stations, Acta Agric. Scand. 30 (1980) 185-188.

[18] McPhee C.P., Selection for efficient lean growth in a pig herd, Aust. J. Agric. Res. 32 (1981) 681-690.

[19] McPhee C.P., MacBeth M., A profit model for estimating economic values of traits in the National Pig Improvement Program, PDRC DAG58/1339 final report, 2000.

[20] McPhee C.P., Trout G.R., The effects of selection for lean growth and the halothane allele on carcass and meat quality of pigs transported long and short distances to slaughter, Livest. Prod. Sci. 42 (1995) 55-62.

[21] McPhee C.P., Rathmell G.A., Daniels L.J., Cameron N.D., Selection in pigs for increased lean growth rate on a time-based feeding scale, Anim. Prod. 47 (1988) 149-156.

[22] Merks J.W.M., Genotype $\times$ environment interactions in pig breeding programmes. II. Environmental effects and genetic parameters in central test, Livest. Prod. Sci. 16 (1987) 215-228.

[23] Merks J.W.M., Donker R.A., Brascamp E.W., Buiting G.A.J., A performance test of pigs according to a modified Kielanowski system, Anim. Prod. 44 (1987) 303-310.

[24] Nguyen H.N., Direct and correlated responses to selection in Large White pigs for growth rate on restricted feeding, Ph.D. Thesis, University of Queensland, Australia, 2002.

[25] Webster A.J.F., Selection for leanness and energetic efficiency of growth in meat animals, Proceeding of Nutrition Society 36 (1977) 53-59.

[26] Woltmann M.D., Clutter A.C., Buchanan D.S., Dolezal H.G., Growth and carcass characteristics of pigs selected for fast or slow gain in relation to feed intake and efficiency, J. Anim. Sci. 70 (1992) 1049-1059.

To access this journal online: www.edpsciences.org 